\title{
Asymmetry Index
}

National Cancer Institute

\section{Source}

National Cancer Institute. Asymmetry Index. NCI Thesaurus. Code C94860.

A normalized measure of a difference between 2 quantities, expressed as a percentage

difference of either their mean or their sum, whichever is defined by a user. 